\title{
Simulação de uma Curva de Titulação Ácido Fraco - Base Forte
}

Fernando M. S. Silva Fernandes Leonel A. T. P. Neves Departamento de Química e CECUL

Faculdade de Ciências de Lisboa

\section{Introdução}

Um dos problemas que se coloca frequentemente na vida prática dum químico é a titulação dum ácido por uma base. Para além do aparato experimental necessário para a experiência, tem de saber-se qual o indicador apropriado para detectar o ponto final da neutralização que se pretende realizar.

Este problema é importante sob o ponto de vista experimental pois a detecção visual do ponto final duma titulação, além de ser uma prática corrente, não implica os erros experimentais que à primeira vista podem ser sugeridos por estar envolvido um aspecto subjectivo $[1,2]$.

O problema resolve-se, experimentalmente, fazendo ensaios prévios com um sistema análogo ao que se pretende analisar, detectando as variaçð̃es bruscas de $\mathrm{pH}$ no ponto de equivalência e, então, procurando numa tabela de indicadores o mais apropriado de acordo com o seu $\mathrm{pK}_{\mathrm{I}}$ e a sua zona de viragem [2].

O objectivo deste trabalho é mostrar que esses ensaios prévios podem ser realizados por um microcomputador sem a necessidade de os executar experimentalmente. Para tal, simula-se a curva de titulação resolvendo as equações que dão o pH duma mistura de ácido fraco e de base forte para diferentes concentraçð̃es do ácido e da base na mistura. Uma vez determinado o $\mathrm{pH}$ no ponto de equivalência, pesquisa-se numa tabela o indicador mais apropriado.

Pode pensar-se que uma metodologia deste género conduz a uma situação semelhante à de já não se saber, em geral, como calcular uma raiz quadrada ou um logaritmo sem a ajuda duma calculadora. Todavia, a situação é diferente, pois o problema subjacente a uma titulação e escolha de indicadores é normalmente tratado a nível teórico com suficiente detalhe. Por outro lado, a possibilidade de gerar curvas de titulação no vídeo do microcomputador, para diferentes sistemas e situações, facilitará certamente a aprendizagem.

\section{Equações Relevantes}

Vamos supor que partimos dum certo volume, VA (em $\mathrm{cm}^{3}$ ), duma solução aquosa de ácido fraco HA (o ácido acético, por exemplo) de concentração molar CA. Daqui podemos determinar o número de moles do ácido existentes no volume VA:

$$
\mathrm{NA}=\mathrm{VA} * \mathrm{CA} / 1000
$$

Pouco a pouco vamos deitando sobre o ácido um certo número de moles, $\mathrm{NB}$, de base forte (o hidróxido de sódio, por exemplo) processo que é controlado pela coordenada da titulação f(i):

$$
\mathrm{NB}=\mathrm{f}(\mathrm{i}) * \mathrm{NA}
$$

Fazemos variar f(i) de 0 a 2 com incrementos de 0.05 o que conduzirá a uma titulação com 41 passos; i variará de 0 a 40 correspondendo o passo 20 ao ponto de equivalência. A partir de $\mathrm{NB}$ e da concentração molar de base, CB, pode calcular-se o volume total da base adicionado ao fim de cada passo:

$$
\mathrm{VB}=1000 * \mathrm{NB} / \mathrm{CB}
$$

e, consequentemente, o volume total da mistura $\mathrm{VT}=\mathrm{VA}+\mathrm{VB}$.

Daqui é imediato o cálculo das concentrações totais do ácido e da base na mistura ao fim de cada passo e supondo que ainda não reagiram: $\mathrm{AT}=1000 * \mathrm{NA} / \mathrm{VT}$ e $\mathrm{BT}=1000 * \mathrm{NB} / \mathrm{VT}$ respectivamente.

Como temos uma titulação com 41 passos teremos uma curva de titulação com 41 valores de $\mathrm{pH}$ que serão armazenados em pH(i).

Antes do ponto de equivalência, isto é, enquanto $0 \leqslant \mathrm{f}<1$ temos a considerar os seguintes equilibrios:

$$
\mathrm{HA}+\mathrm{H}_{2} \mathrm{O} \rightleftarrows \mathrm{A}^{-}+\mathrm{H}_{3} \mathrm{O}^{+}
$$

para o qual se define a constante de acidez:

$$
\begin{aligned}
& \mathrm{KA}=\left[\mathrm{A}^{-}\right]\left[\mathrm{H}_{3} \mathrm{O}^{+}\right] /[\mathrm{HA}] \\
& \mathrm{H}_{2} \mathrm{O}+\mathrm{H}_{2} \mathrm{O} \rightleftarrows \mathrm{H}_{3} \mathrm{O}^{+}+\mathrm{OH}^{-}
\end{aligned}
$$

para o qual se define o produto iónico da água

$$
\mathrm{KW}=\left[\mathrm{H}_{3} \mathrm{O}^{+}\right]\left[\mathrm{OH}^{-}\right]
$$

Como a base é forte considera-se completamente ionizada e os iões $\mathrm{OH}^{-}$dela provenientes reagem com os $\mathrm{H}_{3} \mathrm{O}^{+}$provenientes do ácido.

As espécies electricamente carregadas que existem na solução são: $\mathrm{A}^{-}, \mathrm{H}_{3} \mathrm{O}^{+}, \mathrm{OH}^{-}$e $\mathrm{Na}^{+}$. E fácil concluir que:

$$
\mathrm{AT}=[\mathrm{HA}]_{\mathrm{eq}}+\left[\mathrm{A}^{-}\right]_{\mathrm{eq}}
$$

Por outro lado, a condição de electroneutralidade é:

$$
\mathrm{BT}+\left[\mathrm{H}_{3} \mathrm{O}^{+}\right]_{\mathrm{eq}}=\left[\mathrm{A}^{-}\right]_{\mathrm{eq}}+\left[\mathrm{OH}^{-}\right]_{\mathrm{eq}}
$$

traduzindo o facto do número de cargas positivas ser igual ao número de cargas negativas.

As expressð̃es (4), (5), (6) e (7) constituem as condiçðes 
do problema. A partir delas e por simples manipulação algébrica é fácil eliminar todas as concentrações [ $]_{\text {eq }}$, excepto $\left[\mathrm{H}_{3} \mathrm{O}^{+}\right]_{\mathrm{eq}}$. Tal eliminação conduz à seguinte equação cúbica (que será designada no algoritmo por APE):

$$
\begin{array}{r}
{\left[\mathrm{H}_{3} \mathrm{O}^{+}\right]^{3}+(\mathrm{KA}+\mathrm{BT}) *\left[\mathrm{H}_{3} \mathrm{O}^{+}\right]^{2}+(\mathrm{KA} * \mathrm{BT}-} \\
-\mathrm{KA} * \mathrm{AT}-\mathrm{KW}) *\left[\mathrm{H}_{3} \mathrm{O}^{+}\right]-\mathrm{KA} * \mathrm{KW}=0
\end{array}
$$

em que $\left[\mathrm{H}_{3} \mathrm{O}^{+}\right]$é a incógnita. Todas as outras quantidades que figuram na equação são determinadas a priori.

Resolvendo esta equação para diferentes valores de AT e BT pode calcular-se o $\mathrm{pH}$ da mistura ao longo do processo antes do ponto de equivalência.

No ponto de equivalência e depois, isto é, quando $f \geqslant 1$ teremos:

a) quando $\mathrm{f}=1(\mathrm{NB}=\mathrm{NA})$ formam-se NA moles de sal $\mathrm{NaA}$; é o ponto de equivalência.

b) quando $\mathrm{f}>1$ continuarão a existir NA moles de sal e NB - NA moles de base em excesso.

As concentrações do sal e da base em excesso serão $\mathrm{ST}=1000 * \mathrm{NA} / \mathrm{VT}$ e $\mathrm{BE}=1000 *(\mathrm{NB}-\mathrm{NA}) / \mathrm{VT}$ respectivamente.

O sal, totalmente ionizado, sofrerá hidrólise e a base em excesso considera-se, também, totalmente ionizada. Os equilíbrios presentes serão:

$$
\mathrm{A}^{-}+\mathrm{H}_{2} \mathrm{O} \rightleftarrows \mathrm{HA}+\mathrm{OH}^{-}
$$

para o qual se define a constante de hidrólise:

$$
\mathrm{KH}=[\mathrm{HA}]\left[\mathrm{OH}^{-}\right] /\left[\mathrm{A}^{-}\right]=\mathrm{KW} / \mathrm{KA}
$$

e novamente o equilíbrio da auto-ionização da água com a respectiva constante $\mathrm{KW}$.

Temos a considerar, agora, os seguintes balanços:

$$
\mathrm{ST}=\left[\mathrm{A}^{-}\right]_{\mathrm{eq}}+[\mathrm{HA}]_{\mathrm{eq}}
$$

e

$$
\mathrm{BE}+\mathrm{ST}+\left[\mathrm{H}_{3} \mathrm{O}^{+}\right]_{\mathrm{eq}}=\left[\mathrm{OH}^{-}\right]_{\mathrm{eq}}+\left[\mathrm{A}^{-}\right]_{\mathrm{eq}}
$$

sendo esta última a condição de electroneutralidade.

Subtraindo membro a membro as equações anteriores obtém-se:

$$
[\mathrm{HA}]_{\mathrm{eq}}=\left[\mathrm{OH}^{-}\right]_{\mathrm{eq}}-\left[\mathrm{H}_{3} \mathrm{O}^{+}\right]_{\mathrm{eq}}-\mathrm{BE}
$$

As equaçð̃es (9), (5), (11) e (12) constituem as condiçð̃es do problema. De modo semelhante ao que se fez anteriormente é fácil eliminar entre elas todas as concentraçð̃es [ $]_{\text {eg }}$, excepto $\left[\mathrm{H}_{3} \mathrm{O}^{+}\right]$, obtendo-se a seguinte equação cúbica (que será designada no algoritmo por PEDP):

$$
\begin{aligned}
& {\left[\mathrm{H}_{3} \mathrm{O}^{+}\right]_{+}^{3}\left(\mathrm{BE}+\mathrm{ST}+\frac{\mathrm{KW}}{\mathrm{KH}}\right) *\left[\mathrm{H}_{3} \mathrm{O}^{+}\right]^{2}-(\mathrm{KW}-\mathrm{BE} *} \\
& \left.* \frac{\mathrm{KW}}{\mathrm{KH}}\right) *\left[\mathrm{H}_{3} \mathrm{O}^{+}\right]-\frac{\mathrm{KW}^{2}}{\mathrm{KH}}=\mathrm{O}
\end{aligned}
$$

em que $\left[\mathrm{H}_{3} \mathrm{O}^{+}\right]$é a incógnita. Todas as outras quantidades são também determinadas a priori como na equação (8). Como $\mathrm{KH}=\mathrm{KW} / \mathrm{KA}$ a equação anterior pode ser rescrita na forma:

$$
\begin{aligned}
& {\left[\mathrm{H}_{3} \mathrm{O}^{+}\right]^{3}+(\mathrm{BE}+\mathrm{ST}+\mathrm{KA}) *\left[\mathrm{H}_{3} \mathrm{O}^{+}\right]^{2-}} \\
& -(\mathrm{KW}-\mathrm{BE} * \mathrm{KA}) *\left[\mathrm{H}_{3} \mathrm{O}^{+}\right]-\mathrm{KW} * \mathrm{KA}=\mathrm{O}
\end{aligned}
$$

Resolvendo esta equação para o valor de ST e para diferentes valores de BE pode calcular-se o $\mathrm{pH}$ da mistura ao longo do processo no ponto de equivalência $\mathrm{e}$ após o ponto de equivalência.

\section{Método de Resolução das Equações}

Embora haja métodos analíticos para resolver equações cúbicas [3] as fórmulas resolventes não são tão simples como para as equaçð̃es do $2 .^{\circ}$ grau. Por outro lado, no caso das equações anteriores só estamos interessados na raiz real e positiva. Finalmente, se tivermos equações algébricas de grau igual ou superior a cinco não existem, em geral, fórmulas resolventes. É de toda a conveniência, então, que se estabeleçam métodos numéricos que nos permitam resolver equações de qualquer grau e que sejam simples de implementar num computador.

$\mathrm{O}$ método numérico que utilizamos para resolver as equações (8) e (13) é o método das bissecçð̃es sucessivas. Trata-se de um método sugestivo, simples de compreender e de implementar e, para o caso presente, suficiente rápido. Os leitores interessados encontrarão outros métodos, porventura mais expeditos, nas referências $[4,5]$.

$\mathrm{O}$ método das bissecções sucessivas é um método iterativo que pressupõe o conhecimento prévio dum intervalo $[\mathrm{X} 1, \mathrm{X} 2]$ onde a raiz se encontre. A determinação desse intervalo pode fazer-se, por exemplo, por estudos gráficos prévios ou, então, a partir de considerações fisicas que condicionem o problema.

No caso presente, vejamos que podemos determinar um intervalo por simples consideraçōes físicas. Assim: a) Antes do ponto de equivalência a concentração $\left[\mathrm{H}_{3} \mathrm{O}^{+}\right]$teórica mínima será 0 , pois concentrações negativas não são fisicamente possíveis.

A concentração $\left[\mathrm{H}_{3} \mathrm{O}^{+}\right]$teórica máxima será $\mathrm{AT}+(\mathrm{KW})^{1 / 2}$ que corresponde à ionização total do ácido. Portanto:

$$
\mathrm{X} 1=0 \quad \text { e } \quad \mathrm{X} 2=\mathrm{AT}+(\mathrm{KW})^{1 / 2}
$$

No algoritmo fazemos a identificação $\mathrm{X} \equiv\left[\mathrm{H}_{3} \mathrm{O}^{+}\right]$.

b) No ponto de equivalência e depois é óbvio que:

$\mathrm{X} 1=0 \quad$ e $\quad \mathrm{X} 2=10^{-7}$

Uma vez determinado o intervalo [X1, X2] calcula-se o seu ponto médio:

$$
\mathrm{XM}=(\mathrm{X} 1+\mathrm{X} 2) / 2
$$

e pesquisa-se em qual dos subintervalos [X1, XM] e [XM, X2] a raiz se encontra.

Se a raiz está em $[\mathrm{X} 1, \mathrm{XM}]$ então o novo valor de X2 passará a ser $\mathrm{XM}$ e faremos $\mathrm{X} 2=\mathrm{XM}$. Senão a raiz encontra-se em [XM, X2] e o novo valor de X1 passará a 
ser $\mathrm{XM}$ e faremos $\mathrm{X} 1=\mathrm{XM}$. $\mathrm{O}$ processo é então repetido.

É evidente que pela repetição deste processo podemos aproximar-nos da raiz tanto quanto quisermos porque, em cada passo, reduzimos a metade o intervalo onde a raiz se encontra. Assim, 10 passos reduzirão o intervalo por um factor de $2^{10}$ ou seja cerca de 1000 , enquanto 20 passos reduzirão o intervalo por um factor de $2^{20}$ ou seja cerca de 1000000 .

$O$ processo tem necessariamente de terminar e isso é feito logo que o comprimento do intervalo $|\mathrm{X} 1-\mathrm{X} 2|<\mathrm{PE}$ onde PE é um valor pré-estabelecido e que depende do erro que queiramos estabelecer para o método. No caso presente, uma boa escolha, que os leitores poderão comprovar, será $10^{-6}-10^{-8}$ antes do ponto de equivalência e $10^{-11}-10^{-14}$ no ponto de equivalência e depois.

Resta estabelecer um critério para decidir em que subintervalo a raiz se encontra após cada partição do intervalo [X1, X2]. Tal critério baseia-se no seguinte resultado da análise matemática [4]: se uma função $\mathrm{f}(\mathrm{x})$ tem uma única raiz no intervalo $[\mathrm{X} 1, \mathrm{X} 2]$ então $f(X 1) * f(X 2)<0$. Este resultado é intuitivo se repararmos que quando o gráfico duma função corta o eixo dos $\mathrm{x}$ num ponto (esse ponto será a raiz) a função muda de sinal.

Após cada partição do intervalo analisa-se o valor de $f(X 1) * f(X M)$. Se for positivo então a raiz estará em [XM, X2] e atribui-se o valor de XM a X1. Senão a raiz estará em [X1, XM] a atribui-se XM a X2. É claro que, logicamente, deveremos considerar o caso do valor ser nulo o que corresponderá a XM ser a raiz.

No nosso caso, a função f é a função da expressão (8) que no algoritmo se designa por $\mathrm{APE}(\mathrm{X})$ ou a função das expressões (13) ou (14) que no algoritmo se designa por PEDP(X).

Quando o processo terminar, isto é, quando $\mid \mathrm{X} 1-$ $-\mathrm{X} 2 \mid<\mathrm{PE}$ considera-se a raiz, que no algoritmo será designada por $\mathrm{H}$, como o ponto médio do intervalo, valor que é afectado por um erro máximo de $\mathrm{PE} / 2$. Uma vez determinado o $\mathrm{pH}$ no ponto de equivalência procura-se numa tabela, com NIND indicadores, $o$ indicador cuja zona de viragem contenha $\mathrm{o} \mathrm{pH}$ do ponto de equivalência. A tabela contém os nomes dos indicadores em NOMEIND(i), os respectivos $\mathrm{pK}_{1}$ em PKI(i) e os limites inferior e superior da zona de viragem para cada indicador em LI(i) e LS(i) respectivamente; i varia de 1 a NIND. SeLI(i) $<\mathrm{pH}(20)<\mathrm{LS}(\mathrm{i})$ então o indicador cujo nome está em NOMEIND(i) será apropriado para a titulação considerada.

\section{Algoritmo}

Apresentamos seguidamente o algoritmo para a simulação da curva de titulação dum ácido fraco por uma base forte e para a escolha do indicador apropriado. O algoritmo é representado em notação algorítmica $[6,7]$. Esta notação é um meio-termo entre uma linguagem natural (o português, por exemplo) e uma linguagem de programação particular (o BASIC ou o PASCAL, por exemplo). A sua utilização liberta o leitor do conhecimento duma linguagem de programação particular e das ambiguidades e extensão duma linguagem natural. Por outro lado, a notação algorítmica destaca a lógica subjacente no algoritmo. Dado que não se trata duma linguagem, não tem regras sintáticas rígidas e os leitores são livres de representar as operações primitivas da forma que mais lhes agradar. A única condi- ção é que a forma escolhida seja sucinta, clara e não tenha ambiguidades. Como a notação algorítmica é suficientemente clara não faremos quaisquer considerações detalhadas sobre ela. Estamos certos que os leitores se aperceberão das suas particularidades e vantagens ao longo da leitura do algoritmo. Este é constituído por uma parte principal da qual é chamada a subrotina RAIZ que resolve as equações que referimos atrás. A subrotina e as funçð̄es encontram-se descritas após o algoritmo principal.

A codificação do algoritmo numa linguagem de programação particular, conduzindo ao programa, é um passo rotineiro que nada tem a ver com a lógica subjacente, mas apenas com as regras sintáticas da linguagem escolhida.

Posto isto, passemos ao algoritmo. Tudo o que se encontra entre chavetas são apenas comentários.

\section{ALGORITMO PRINCIPAL}

\{Leitura do número e tabela de indicadores\}. Ler NIND

Para $\mathrm{i}$ variando de 1 a NIND repetir Ler NOMEIND (i), PKI(i), LI(i), LS(i)

\{Leitura de variáveis\}

Ler VA, CA, CB, KA, PE

$\mathrm{KW} \leftarrow 1.0 \mathrm{E}-14$

$\mathrm{NA} \leftarrow \mathrm{VA} * \mathrm{CA} / 1000$

Escrever VA, CA, CB, KA, KW, PE

\{Antes do ponto de equivalência\}

Para i variando de 0 a 19 repetir

$$
\begin{aligned}
& f(i) \leftarrow i * 5 / 100 \\
& \mathrm{NB} \leftarrow \mathrm{f}(\mathrm{i}) * \mathrm{NA} \\
& \mathrm{VB} \leftarrow 1000 * \mathrm{NB} / \mathrm{CB} \\
& \mathrm{VT} \leftarrow \mathrm{VA}+\mathrm{VB} \\
& \mathrm{AT} \leftarrow 1000 * \mathrm{NA} / \mathrm{VT} \\
& \mathrm{BT} \leftarrow 1000 * \mathrm{NB} / \mathrm{VT} \\
& \text { \{Parâmetros de subrotina RAIZ\} } \\
& \mathrm{X} 1 \leftarrow 0 \\
& \mathrm{X} 2 \leftarrow \mathbf{A T}+\sqrt{\mathrm{K}} \overline{\mathrm{W}} \\
& \text { BAND } \leftarrow 0 \text { \{BAND }=0 \text { indica função } \mathrm{APE}(\mathrm{X}) \text {; } \\
& \mathrm{BAND}=1 \text { a função } \operatorname{PEDP}(\mathrm{X})
\end{aligned}
$$

CHAMAR SUBROTINA RAIZ

Escrever AT, BT, H, ERRO, f(i), pH(i)

\{Ponto de equivalência e depois\}

Ler PE

Para i variando de 20 a 40 repetir

$f(i) \leftarrow i * 5 / 100$

$\mathrm{NB} \leftarrow \mathrm{f}(\mathrm{i}) * \mathrm{NA}$

$\mathrm{VB} \leftarrow 1000 * \mathrm{NB} / \mathrm{CB}$

$\mathrm{VT} \leftarrow \mathrm{VA}+\mathrm{VB}$

$\mathrm{ST} \leftarrow 1000 * \mathrm{NA} / \mathrm{VT}$

$\mathrm{BE} \leftarrow 1000 *$ (NB-NA)/VT

\{Parâmetros de subrotina RAIZ\}

$\mathrm{X} 1 \leftarrow 0$

$\mathrm{X} 2 \leftarrow 1.0 \mathrm{E}-7$

BAND $\leftarrow 1$ 


\section{CHAMAR SUBROTINA RAIZ}

Escrever ST, BE, H, ERRO, f(i), pH(i)

\{Representação Gráfica dos valores Calculados\} Para i variando de 0 a 40 repetir

PONTO (f(i) * FH, pH(i) * FV)

\{PONTO é uma rotina gráfica que escreve um pixel no ponto $(\mathrm{x}, \mathrm{y})$ do vídeo: $\mathrm{FH}$ e $\mathrm{FV}$ são os

factores de escala\}

\{Escolha do Indicador\}

$\mathrm{SENT} \leftarrow 0$ [Se SENT permanecer com o valor 0 não há indicador que sirva\}

Para i variando de 1 a NIND repetir

Se (LI (i) $<$ pH $(20)<$ LS (i) então

Escrever NOMEIND(i), PKI(i), LI(i), LS(i), $\mathrm{pH}(20)$

SENT $\leftarrow 1$

$\mathrm{Se} \mathrm{SENT}=0$ então

Escrever "A lista dos indicadores não contém um indicador apropriado para a titulação",

\section{FIM DO ALGORITMO PRINCIPAL}

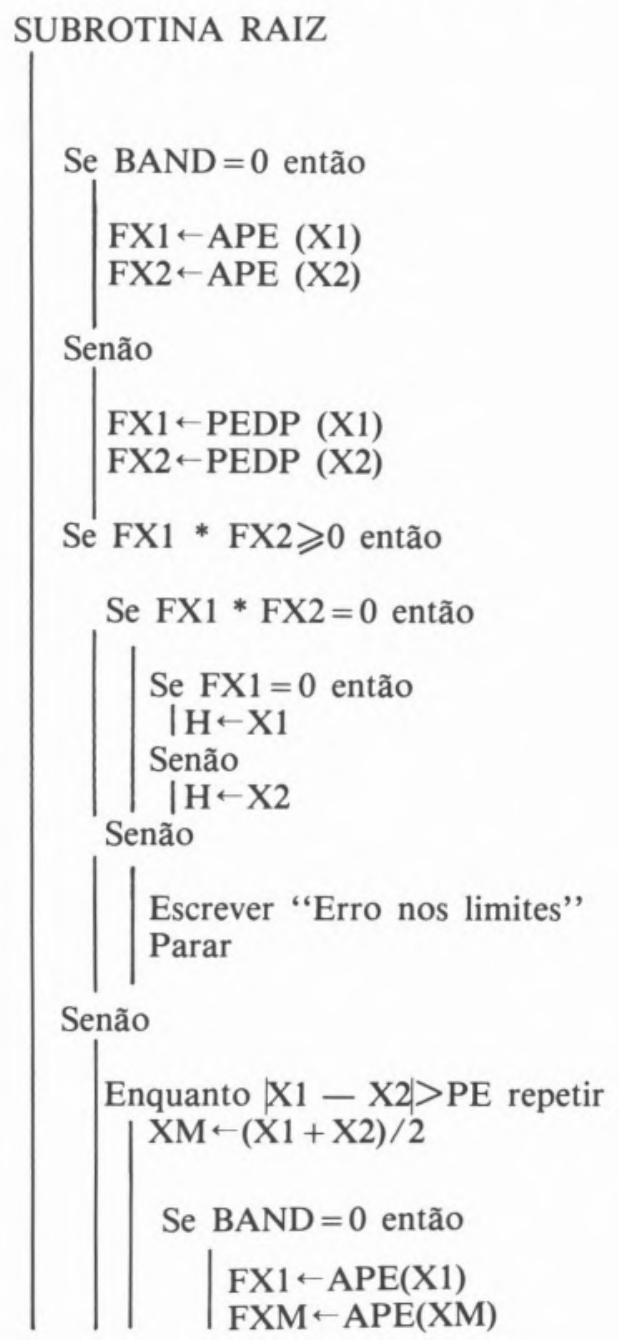

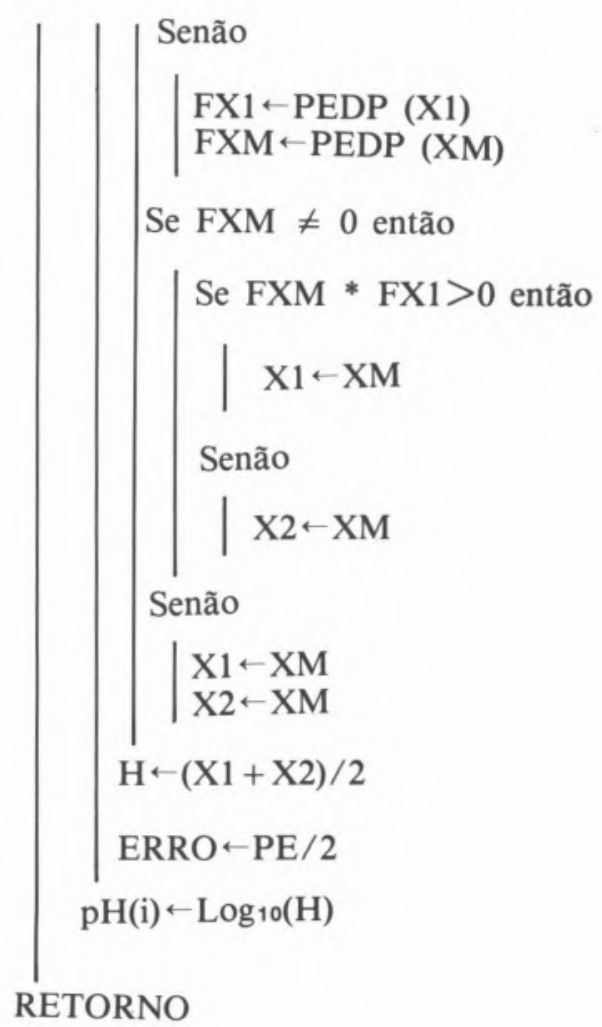

FUNÇÃO APE (X)

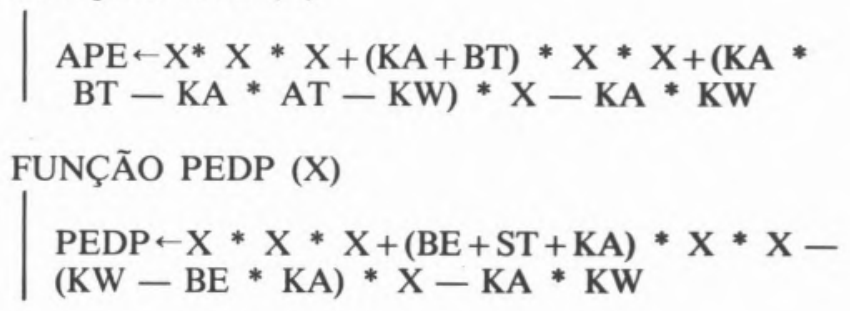

Muitos microcomputadores com capacidade gráfica têm como primitiva, uma rotina semelhante à rotina PONTO do nosso algoritmo, bastando invocá-la no programa. Essa invocação é feita normalmente através da instrução PLOT $(x, y)$. Contudo, se um microcomputador com capacidade gráfica não tiver, como primitiva, uma rotina semelhante a PONTO terá certamente outras primitivas gráficas a partir das quais o utilizador poderá construir uma rotina PONTO.

Queremos sublinhar que um algoritmo traduz um padrão de comportamento sem referência a um processador particular. Um programa, no entanto, tem especificaçð̃es detalhadas com respeito a um processador lógico particular. Isto significa que os leitores que pretendam codificar o algoritmo anterior devem estar alertados para o facto de certos microcomputadores não aceitarem, por exemplo, variáveis indexadas com mais de um símbolo para os seus identificadores. No nosso algoritmo os valores de $\mathrm{pH}$ ao longo do processo são armazenados em pH(i). Alguns microcomputadores não aceitam este identificador pelo facto de ter dois símbolos. Nesse caso pode, por exemplo, substituir-se $\mathrm{pH}(\mathrm{i})$ por $\mathrm{p}(\mathrm{i})$. 
Por outro lado, as primitivas:

Se (condição) então

acção 1

Senão

acção 2

e

Enquanto (condição) repetir acção

que utilizámos ao longo do algoritmo podem não ter correspondentes directos em algumas linguagens de programação. De facto, as estruturas IF/THEN/ELSE e WHILE não existem ainda em todas as versð̃es das linguagens de alto nível. Todavia é possível construir estruturas semelhantes com as instruções IF/THEN e GOTO as quais existem em todas as linguagens mais comuns. Mas isto são problemas sintáticos das linguagens de programação que podem ser facilmente resolvidos com o manual da linguagem utilizada e se tivermos, à partida, um algoritmo representado numa notação algorítmica clara.

\section{Resultados}

Na tabela 1 apresentamos alguns dos resultados de simulação duma neutralização de $50 \mathrm{~cm}^{3}$ de uma solução aquosa de ácido acético $0.1 \mathrm{M}$ por uma solução aquosa de hidróxido de sódio $0.1 \mathrm{M}$.

\section{Tabela 1}

Resultados da Simulação de uma Titulação de Ácido Acético $0.1 \mathrm{M}$ com Hidróxido de Sódio $0.1 \mathrm{M}$

$\begin{array}{lc}\text { f } & \text { pH } \\ 0 & 2.87 \\ 0.1 & 3.79 \\ 0.2 & 4.13 \\ 0.3 & 4.36 \\ 0.4 & 4.56 \\ 0.5 & 4.73 \\ 0.6 & 4.91 \\ 0.7 & 5.10 \\ 0.8 & 5.33 \\ 0.9 & 5.67 \\ 1.0 & 8.75 \\ 1.1 & 11.76 \\ 1.2 & 12.05 \\ 1.3 & 12.20 \\ 1.4 & 12.32 \\ 1.5 & 12.39 \\ 1.6 & 12.41 \\ 1.7 & 12.50 \\ 1.8 & 12.54 \\ 1.95 & 12.59\end{array}$

Para o processo antes do ponto de equivalência assumimos um erro de iteração de $10^{-8}$ o que implicou cerca de 20 iterações por passo; no ponto de equivalência, e dépois, assumimos um erro de $10^{-14}$ o que implicou cerca de $\mathbf{4 0}$ iterações por passo. No total, foram realizadas cerca de 1200 iterações.

O tempo de execução depende evidentemente do microcomputador e da linguagem utilizados. Codificando o algoritmo anterior em BASIC, o respectivo programa executado num microcomputador de pequeno porte (o
ZX Spectrum, por exemplo) consumirá cerca de $15 \mathrm{~min}$ para construir a curva de titulação, desenhá-la e escolher o indicador. Num microcomputador de 16 bits (o ai - M16 ou o QL, por exemplo) o tempo será substancialmente reduzido, em particular, se a codificação do algoritmo for feita em PASCAL: 2 min quando muito. Na Fig. 1 representa-se a cópia da curva de titulação desenhada no vídeo. $\mathrm{O}$ indicador escolhido foi a fenolftaleína como era de esperar.

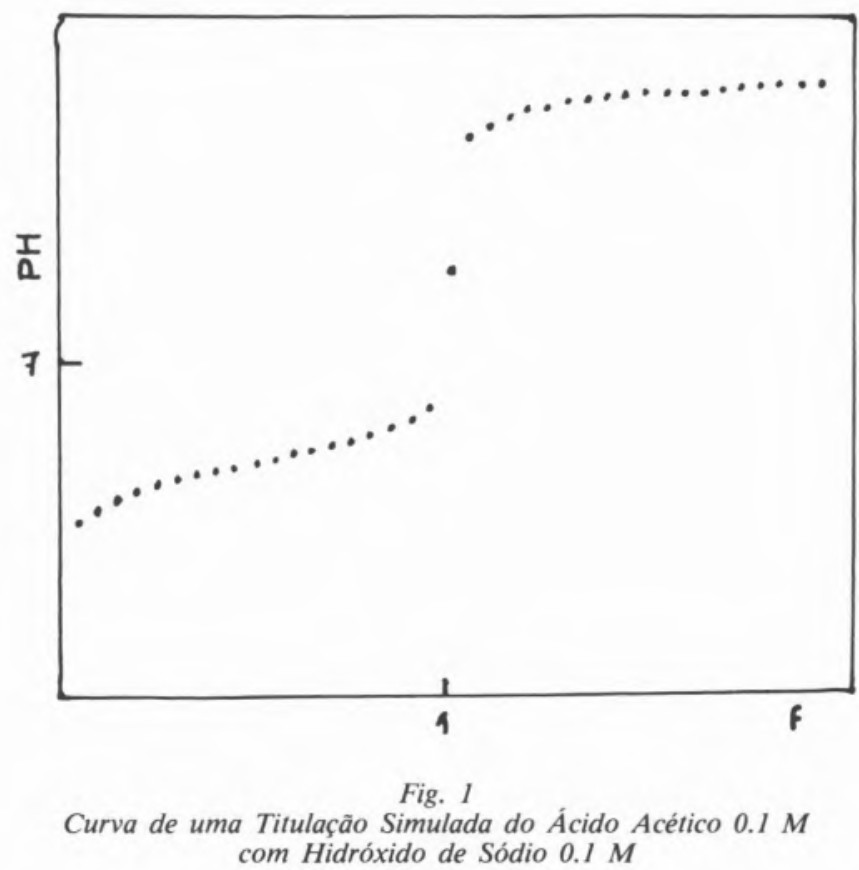

\section{Conclusões}

Os resultados obtidos mostram que é perfeitamente exequível utilizar um microcomputador para delinear uma titulação e escolher o indicador apropriado. Os erros envolvidos são comparáveis, ou mesmo inferiores, aos erros experimentais.

A única aproximação envolvida nas equações que utilizámos é a introdução de concentrações em vez de actividades. Tal aproximação, que é perfeitamente justificável se as concentraçð̃es forem suficientemente baixas pode, no entanto, ser facilmente remediada se as condiçðes do problema o exigirem.

Por outro lado o algoritmo apresentado contempla apenas o caso de ácidos e bases monofuncionais. A sua adaptação ao caso geral de ácidos e bases polifuncionais não é difícil e deixamos essa adaptação ao critério dos leitores.

$\mathrm{O}$ uso da notação algorítmica parece-nos bastante importante pois facilita a compreensão do método e a codificação do algoritmo.

Finalmente, manifestamos aos leitores a nossa disponibilidade para o esclarecimento de quaisquer dúvidas que possam surgir na leitura deste trabalho. 


\section{REFERÊNCIAS}

1. "University Chemistry”, B. Mahan, 3. a Edição, Addison-Wesley, 1975.

2. "Equilibrio Químico e Reacçðes de Oxi-Redução", Fernando Fernandes, Emilia Carvalho e Helena Mendonça, Escölar Editora, 1982. 3. "Mathematical Techniques in Chemistry", J. P. Dence; John Wiley e Sons, 1975.
4. "Elementary Numerical Analysis", Conte e de Boor; McGraw-Hill, 2. ${ }^{a}$ Edição, 1972.

5. "Numerical Methods in Chemistry", K. Jeffrey Johnson, Marcel Dekker, Inc., 1980.

6. "An Introduction to Data Structures with Applications", J. P. Tremblay e P. Sorenson, 2. " Edição, McGraw-Hill, 1984.

7. "Introduction a la Programmation Structurée", Micro-Systems, 117, Maio-Junho, 1982. 